\title{
Synthesis of Fluorescent Carbon Dots by Gastrointestinal Fluid Treatment of Mongolia Har Gabur
}

\author{
Tegexibaiyin Wang, ${ }^{1}$ Wen Zhang, ${ }^{1}$ Xiaofeng Zhang, \\ Xilin Qiqige, ${ }^{1}$ Wuhan Qimuge, ${ }_{1}^{1}$ Aodeng Qimuge, ${ }^{1}$ Hairong Wang, \\ Bulin Baila, ${ }^{1}$ Alateng Qimuge, ${ }^{1}$ Ming Yuan, ${ }^{2}$ and Jun $\mathrm{Ai}^{3}$ \\ ${ }^{1}$ Pharmacy Laboratory, Inner Mongolia International Mongolian Hospital, 83 Daxuedong Road, Hohhot 010065, China \\ ${ }^{2}$ Agricultural Nanocenter, School of Life Sciences, Inner Mongolia Agricultural University, 306 Zhaowuda Road, Hohhot 010018, China \\ ${ }^{3}$ College of Chemistry and Environmental Science, Inner Mongolia Normal University, 81 Zhaowuda Road, Hohhot 010022, China
}

Correspondence should be addressed to Tegexibaiyin Wang; tegexibaiyin@yeah.net

Received 30 January 2017; Revised 6 April 2017; Accepted 24 April 2017; Published 9 July 2017

Academic Editor: Jian Zhong

Copyright (C) 2017 Tegexibaiyin Wang et al. This is an open access article distributed under the Creative Commons Attribution License, which permits unrestricted use, distribution, and reproduction in any medium, provided the original work is properly cited.

\begin{abstract}
Har Gabur is the carbide obtained from pig manure by burning. The fluorescent carbon dots (CDs) of Har Gabur were successfully synthesized through simulating the digestion process of human gastrointestinal tract. Transmission Electron Microscope (TEM) analysis showed that the average size of the prepared Har Gabur CDs was $4 \mathrm{~nm}$, with good solubility in water and strong fluorescence under UV irradiation. The X-ray and Raman results showed that the Har Gabur CDs were mainly composed of oxygen "O" and carbon "C" elements, in the forms of " $\mathrm{C}=\mathrm{O}$ " and "C-O." The bond energy results showed that the nitrogen " $\mathrm{N}$ " atom presented as "C-N" form, which indicated that Har Gabur CDs also contain "N." In photobleaching assay, Har Gabur CDs showed excellent light stability compared with ordinary organic dye, fluorescein, and Rhodamine B. The fluorescence intensity of Har Gabur CDs was fairly stable within a wide $\mathrm{pH}$ range of 3-10. When L-lysine and L-cysteine were applied for the passivation stage, the relative quantum yields were improved by 1.53 and 3.68 times, respectively. Finally, the fluorescence properties of Har Gabur CDs were tested in cells and zebrafish, illustrating that Har Gabur CD has potential in the application of biological labeling and imaging.
\end{abstract}

\section{Introduction}

As one of the most important natural elements, carbon is not only involved in human body but also found to play critical role in the field of medicine [1]. For instance, the charcoal ash is a typical carbonized traditional Mongolian medicine [2]. As a matter of fact, the carbonized medicine is made up of carbon. The traditional Mongolian medicine applies anaerobic burning of a variety of drugs to get the biological carbon powder. The medicines made by these carbon powders are finally ingested through gastrointestinal tracts to function. Clinically, carbonized traditional Mongolian medicine is used for the treatment of esophageal diseases, gastrointestinal diseases, and tumors due to its significant effect. In recent years, the carbon molecules including the most famous molecules fullerene, grapheme, and CD have become the topics of innovative scientific research $[3,4]$.

The CD is also called carbon quantum dot (CQD). It is mainly made up of nanocarbon particles of grapheme quantum dots (GQDs) and CQDs with diameter smaller than $10 \mathrm{~nm}$. The discovery of CDs is mainly through the purification of the single layer nanotube used in early preparative electrophoresis assay and the laser ablation of graphite powder and cement. The diversity of composition and structure of CDs define their different properties. The carboxyl groups on the surface of CDs enable them to have excellent water solubility and biocompatibility. In addition, the physical properties of CDs can be enhanced by chemically modifying the surface structure, for example, by applying a 


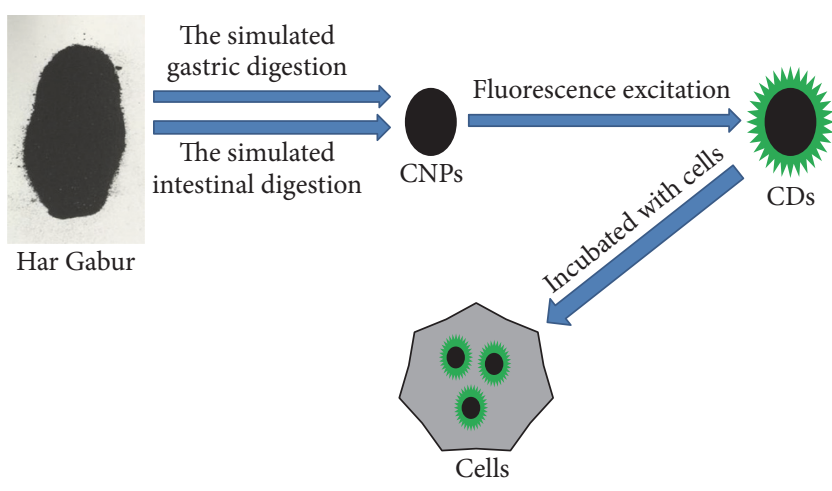

SCHEME 1: Synthesis scheme of carbon dots derived from Har Gabur.

variety of organic, inorganic, and even biological molecules [5-7].

As an innovative fluorescence nanomaterial, CDs have palmary photoluminescence, chemical inertness, and biocompatibility $[8,9]$. Based on the previous studies, CDs would cause no abnormality, damage, and toxicity to tissues, organs, and even genes. Furthermore, the unique optic and biological features of CD give it outstanding advantages and prominent future on the optic imaging, tumor diagnosis, and treatment $[2,8,10]$. There are several kinds of raw materials for CDs, such as ginger, straw, coffee, egg, candle ash, and grapheme [11, 12]. The CDs prepared have been found to have good inhibitory effects on the growth of and antioxidation of hepatocellular carcinoma, lung cancer, breast cancer, and human cervical cancer $[2,13]$.

The preparation methods of CDs have been developed and summarized into several particular refined methodologies including the synthetic, electrochemical, laser ablation, acid dissolution, and hydrothermal method $[14,15]$. In this study, an innovative biological method for CDs preparation has been discussed. This method has a close correlation with the absorption and digestion process of traditional charcoal medicine in human body [16]. In addition, this method could possibly improve the pharmacological effects of CDs [17]. Based on this enzymatic method, the surface of nanostructured CDs is full of organic groups, which enable CDs to have good dispersibility and water solubility $[18,19]$. Moreover, the structure of the CDs obtained through this way would benefit the functional modification and biological coupling labeling that allow medicine to have good biocompatibility.

In this study, Har Gabur CD was used for the first time as a raw material for CD preparation due to its characteristics of simple composition and cheap price. In this study, we simulate the digestion process in human body through mixing Har Gabur CDs with biological enzyme in vitro. These CDs have better water solubility, fluorescence characteristics, and biocompatibility (Scheme 1). Study on Har Gabur CDs not only has great significance for the preparation method of the fluorescent CDs but also has important research value on understanding the physiological mechanism of Har Gabur CDs. In addition to its foundation build up, it has profound significance on the application of drug delivery and targeting.

\section{Materials and Methods}

2.1. Materials. Muffle furnace (Beijing Cinit Electric Co., China) and U-3010 UV-visible spectrophotometer (Hitachi, Japan) were applied to the absorption measurement of carbon solution. F-2500 fluorescence spectrophotometer (Hitachi, Japan) was used for measuring fluorescence excitation and emission spectra. Zetasizer Nano (Malvern Instruments Ltd., UK) was applied for carbon hydrate particle size and surface charge determination. X-ray photoelectron spectroscopy ESCALAB 250X (Thermo, USA) was used for the determination of $C D$ composition and $C$ is spectrum. SpectrumGX infrared transform spectroscopy (PerkinElmer, America) and LabRAMHR800 Raman spectrometer (Joe Pingyi Feng company, France) were used to characterize the surface structure and other characteristics of carbon dots; fluorescence lifetime points by FL-TCSPC (fluorescence spectrometer Joe Feng Pingyi company, France) were measured; PHS-3C (Chengdu ark Technical Developing Company, China) was used for the acidity measurement.

\subsection{Preparation of $C D$}

2.2.1. Preparation of Gastric Liquid. $0.2 \mathrm{~g}$ of $\mathrm{NaCl}, 0.32 \mathrm{~g}$ of pepsin (2330 U/mg prot, Sigma), and $0.7 \mathrm{ml}$ concentrated hydrochloric acid were dissolved in distilled water up to $100 \mathrm{ml}$ after adjusting $\mathrm{pH}$ to 1.2 .

2.2.2. Preparation of Intestinal Liquid. The intestinal tract liquid was prepared by dissolving $0.68 \mathrm{~g}$ of $\mathrm{KH}_{2} \mathrm{PO}_{4}$ into $25 \mathrm{ml}$ distilled water. $\mathrm{NaOH}$ was applied for $\mathrm{pH}$ adjustment ( $\mathrm{pH} 7.5$ ) and additional $19 \mathrm{ml}$ distilled water was added. Then, $1 \mathrm{~g}$ of trypsin (10010 U/mg prot, Sigma) was added, and $\mathrm{NaOH}$ was applied again. Distilled water was added up to $100 \mathrm{ml}$ finally.

\subsubsection{Preparation of Fluorescent CD from Har Gabur CD} Powder. $1.0 \mathrm{~g}$ of Har Gabur CD powder was added to a falcon flask containing prepared simulated gastric acid liquid. The falcon flask was set in the $37^{\circ} \mathrm{C}$ oil bath pot, allowing magnetic stirring for $12 \mathrm{hrs}$. Then, the nonreacted substances were collected through centrifugation. The supernatant was transferred into a dialysis bag (USA Viskase MWCO 1000D) and left in distilled water for 2-3 days, by changing the water once in $1 \mathrm{~h}$ until the $\mathrm{pH}$ reached neutral. After dialysis, the liquid was centrifuged at $1.6 \times 10^{2} \mathrm{rpm}$ for $30 \mathrm{~min}$ and the supernatant was stored at $4^{\circ} \mathrm{C}$. The pellets collected through gastric acid digestion were then recycled for $\mathrm{CD}$ particles. They were mixed with distilled water and centrifuged for $10 \mathrm{~min}$ at $1.6 \times 10^{4} \mathrm{rpm}$, and this step was repeated twice. The pellets were mixed with the simulated intestinal acid liquid and the flask was left in the oil bath for $12 \mathrm{hrs}$ stirring. After reaction, the supernatant was repeatedly done as the above method, putting into a dialysis bag for dialysis reaction and the final liquid was stored at $4^{\circ} \mathrm{C}$.

The collected supernatants from either gastric or intestinal acid liquid treatment were mixed and condensed to $25 \mathrm{ml}$. Then, the condensed liquid was microwaved for $5 \mathrm{~min}$ at 


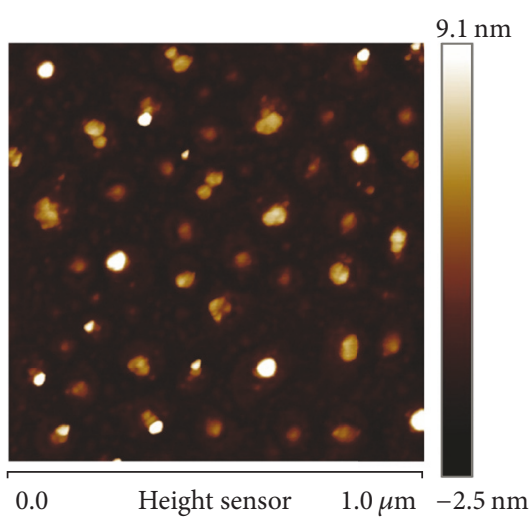

(a)

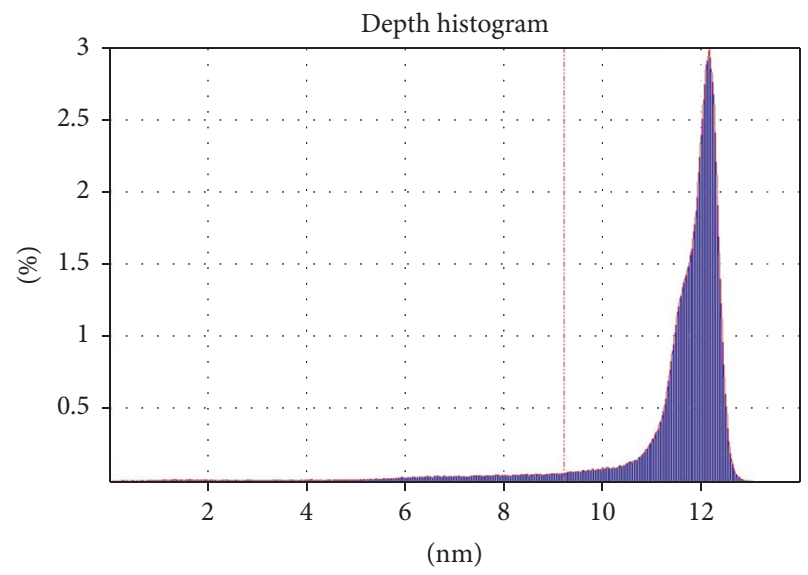

(b)

FIGURE 1: Direct acquisition of 3D image of C-dots by atomic force microscopy. (a) The AFM height mode image of C-dots obtained in air, the upper and bottom bars indicate the dots' height and scale, respectively. (b) The AFM cross-sectional analysis of (a). An average hydrodynamic size of $3.6 \pm 0.102 \mathrm{~nm}$ with a PDI of 0.422 was obtained from the DLS measurement.

$50 \mathrm{~Hz}$. The liquid was then freeze dried and the certain amount of distilled water was added to dissolve it. The final CDs were collected through 1 min centrifugation and sent for fluorescence activity detection or stored at $4^{\circ} \mathrm{C}$.

2.3. Surface Passivation of CD. $100 \mathrm{ml}$ of prepared CDs liquid $(0.5 \mathrm{mg} / \mathrm{ml})$ was mixed with $1 \mathrm{~g}$ surface passivation agent ( $\mathrm{pH} 7.25$ ) and sent for ultrasonic treatment for $5 \mathrm{~min}$. The mixture was heated up to $110^{\circ} \mathrm{C}$ and polylysine (PLL) and cysteine were applied for CDs surface passivation through removal of extra polylysine (PLL) and cysteine and keeping molecules of $500 \mathrm{Da}$ inside of dialysis bag. Passivation using PLL was achieved through filtration. Passivation based on chitosan was also done by filtration and the extra chitosan was removed by precipitation in ethanol.

2.4. Characterizations. Transmission electron microscopy (TEM) was performed using a FEI Tecnai $\mathrm{G}^{2}$ Spirit at an acceleration voltage of $120 \mathrm{kV}$. Nano-ZS90 Zetasizer (Malvern Instruments, Malvern, UK) was used to determine the zeta potential. Absorption and fluorescence spectra were recorded at room temperature on UV-2550 UV-Vis spectrophotometry (Shimadzu, Japan) and Luminescence Spectrometer 55 (PerkinElmer), respectively. XPS spectra were used to characterize the chemical composition using an Escalab $250 \mathrm{Xi}$ X-ray photoelectron spectrometer (Thermo Scientific).

2.5. Fluorescence Quantum Yield Measurements. The fluorescence quantum yield was measured and calculated based on previous studies $[19,20] . \Phi_{x}=\Phi_{\text {std }} I_{x} A_{\text {std }} \eta^{2}{ }_{x} / I_{\text {std }} A_{x} \eta_{\text {std }}^{2}$ was applied and $X$ and $l$ std were the fluorescence strength of CDs and control. $A_{x}$ and $A_{\text {std }}$ were the optical density of CDs and control. The absorbencies of all the samples in $1.0 \mathrm{~cm}$ cuvette were kept under 0.1 at the excitation wavelength to minimize reabsorption effects.
2.6. Cell Experiments. HepG2 cells were inoculated in 24-well culture plate (1000 cells/well). After $12 \mathrm{~h}, 1 \mathrm{mg} / \mathrm{ml}$ of prepared CDs was added to cells and cells were cultivated for extra $2 \mathrm{~h}$. PBS was applied to wash cells and formalin was used for cell fixing. Cell imaging was performed through Nikon $\mathrm{Ti}-\mathrm{U}$ inverted fluorescence microscope, and the excitation wavelengths were $380-420 \mathrm{~nm}$ and $450-490 \mathrm{~nm}$ for blue and green color, respectively $[21,22]$.

2.7. Ex Vivo Zebrafish Experiment. Zebrafish was raised in a standardized light cycled system with a cycle of $14 \mathrm{~h}$ light and $10 \mathrm{~h}$ dark at $28^{\circ} \mathrm{C}$. The imaging system for zebrafish was performed using Cri Maestro. The adult fish was cultivated into a water system with CDs $(5 \mathrm{mg} / \mathrm{ml})$ for $10 \mathrm{~min}$ and washed with distilled water for imaging.

\section{Results and Discussion}

3.1. Size and Zeta Potential. The 3D image of CDs was obtained by atomic force microscopy (Atomic Force, AFM) (Figure 1(a)). An average hydrodynamic size of $3.6 \pm 0.102 \mathrm{~nm}$ with a PDI of 0.422 was obtained from the Dynamic Light Scattering (DLS) measurement. By contrast, the crosssectional analysis of the AFM image performed in air revealed a typical topographic height of approximately $1.40 \mathrm{~nm}$, which is less than that found by the DLS results, because of the AFM imaging mode in air. According to the analysis of the image, we can get the three-dimensional size of the particle (Figure 1(b)). The CDs were evaluated by DLS and atomic force microscopy image analysis for the first time in our study.

3.2. Optical Property. UV-Vis absorption and photoluminescence (PL) emission spectra for CDs were examined. Figure 2(a) shows the detection of CDs surface passivation applying UC spectroscopy and photoluminescence spectroscopy. Fluorescence image of the CD labeled image, its control, 


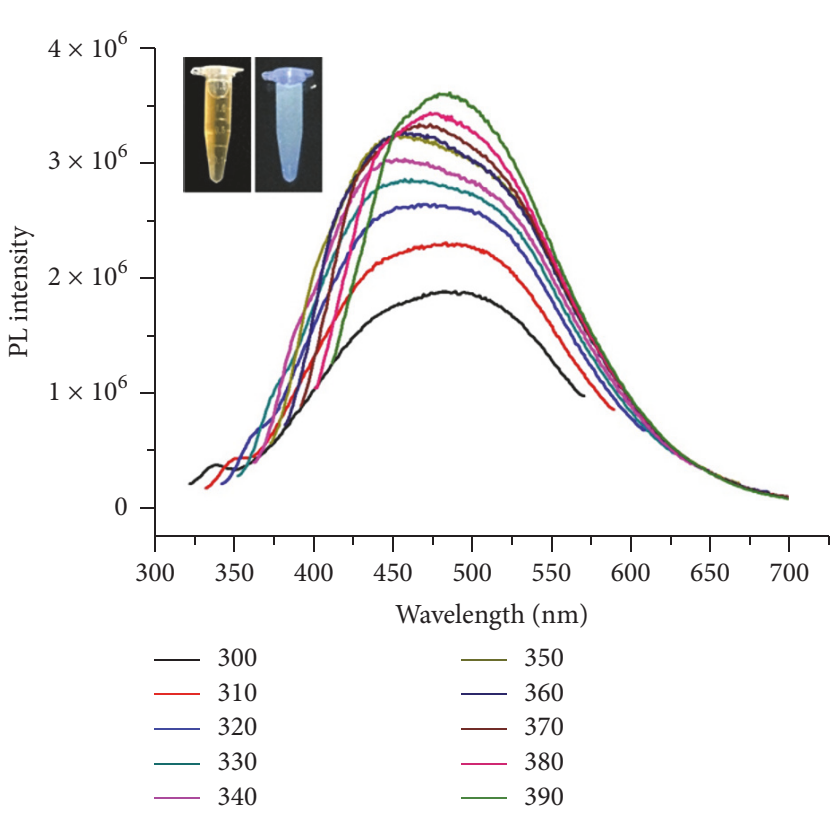

(a)
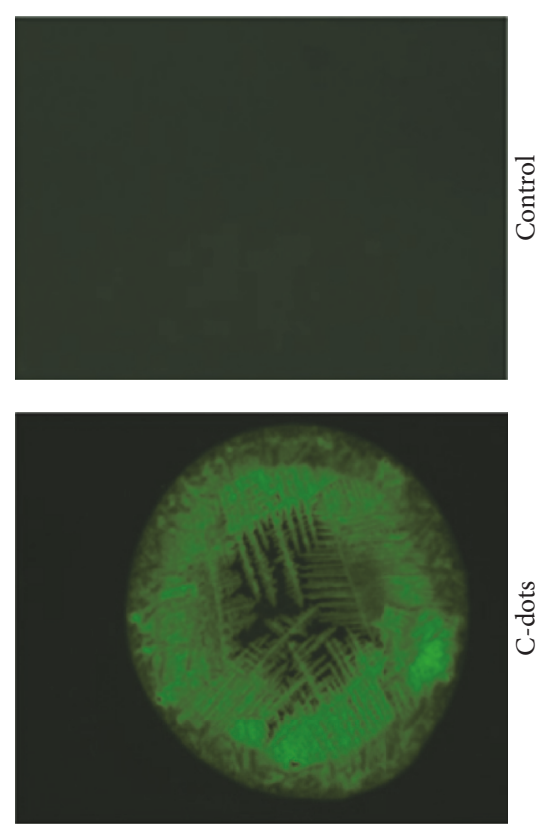

(b)

FIgURE 2: UV-Vis absorption and photoluminescence (PL) emission spectra for C-dots. (a): photograph of the C-dots excited by a UV lamp $(365 \mathrm{~nm})$. PL emission spectra (excitation wavelength from $325 \mathrm{~nm}$ to $445 \mathrm{~nm}$ in $20 \mathrm{~nm}$ increments). (b) Fluorescence image of the C-dot labeled image, its control, and bare C-dots measured with fluorescence microscope imaging system.

and bare CDs were measured with fluorescence microscope imaging system (Figure 2(b)). The one with applying UV spec did not show obvious peaks, which are consistent with the commercial CDs. CQDs corresponding emissions covering the ultraviolet wavelength range from ultraviolet $(\sim 430 \mathrm{~nm})$ yellow to green $(\sim 550 \mathrm{~nm})$. The position of CDs emission shifts from the peak to longer wavelength and the intensity increases with the gradually decreased excitation wavelength. By selecting quinine sulfate in $0.1 \mathrm{M} \mathrm{H}_{2} \mathrm{SO}_{4}$ as the standard, the measured relative fluorescence quantum yield (QY) of the bare CDs was $0.72 \%$. This value was equal to the value of the carbon point from the candle soot and was greater than the value of the CDs from the soot of the natural gas. The luminescence mechanism of CDs is still not clearly understood, and the proposed reason may be radiative recombination. The green luminescence of CDs can be attributed to the surface energy trap, and the blue emission of CDs can be attributed to the $Z$ locus, because of the graphene molecules embedded in the CDs.

3.3. Structure and Constituents. In order to obtain detailed structural and compositional information on the prepared carbon points, we also measured their X-ray diffraction (XRD) and X-ray photoelectron spectroscopy (XPS). XRD data showed that our CDs were amorphous, similar to that reported in other reports. As the wide spectrum XPS scanning shown in Figure 3, 284.74 represents SP2 hybrid graphite. The 288.09 and 286.25 represent " $\mathrm{C}=\mathrm{O}$ " and " $\mathrm{C}-\mathrm{O}$ " bonds, which are the two representatives of graphene oxide structure (Figure 3(b)). The samples mainly contain "C" and "O" elements (Figure 3(a)). Linking this result with "Cls" peak analysis, the samples were composed of graphene and graphene oxide (GO). According to the binding energy, the "N" atom presented in the form of "C-N." Our result proved that the graphite in the sample contains some nitrogen doped graphene (NG). According to the binding energy, the " $\mathrm{Si}$ " atom presented in the form of "Si-O" bond, while "P" and "Na" existed in the form of $\mathrm{NaH}_{2} \mathrm{PO}_{4}$ (Figure 3(b)).

Three strong peaks at 285.4, 400, and $532.5 \mathrm{eV}$ were attributed to oxygen, nitrogen, and carbon. Therefore, the main elements of the prepared CQDs are "C" (60.8\%), "O" (25\%), and "N" (7.2\%) (see Table 1). Cls spectrum (Figure 3(b)) shows three peaks at 284.9, 286, and $288.9 \mathrm{eV}$, which were attributed to "C-C," "C=O," and "C-N." XPS and Raman spectra showed that the "O" and " $\mathrm{C}$ " elements were the main elements in the sample. The "O/C" atoms were in " $\mathrm{C}=\mathrm{O}$ " and " $\mathrm{C}-\mathrm{O}$ " forms, which are two representative graphene oxide (GO) structures (Figure $3(\mathrm{c})$ ).

3.4. Cell Imaging. The potential applications of the fluorescent CDs derived from plant soot were evaluated in HepG2 cells imaging. The HepG2 cells incubated with PLLpassivated CDs in Figure 4 become bright when existing at the wavelength of $450-490 \mathrm{~nm}$. This indicates that PLL passivation of HepG2 can be labeled CDs cells in a simple incubator. In addition, no quenching effect was observed in the continuous excitation of cell imaging in 15 minutes.

3.5. Ex Vivo Zebrafish Imaging. For organism imaging, we chose a small guppy, which is one of the vertebrates which can be used in Maestro imaging system to observe the 


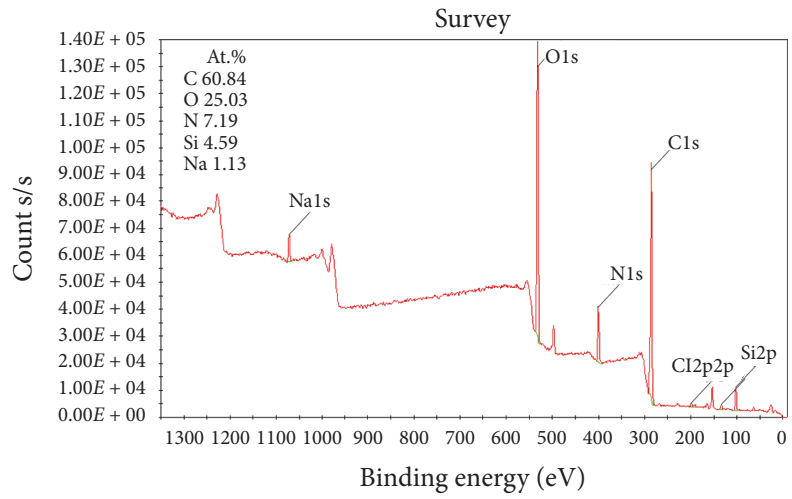

(a)

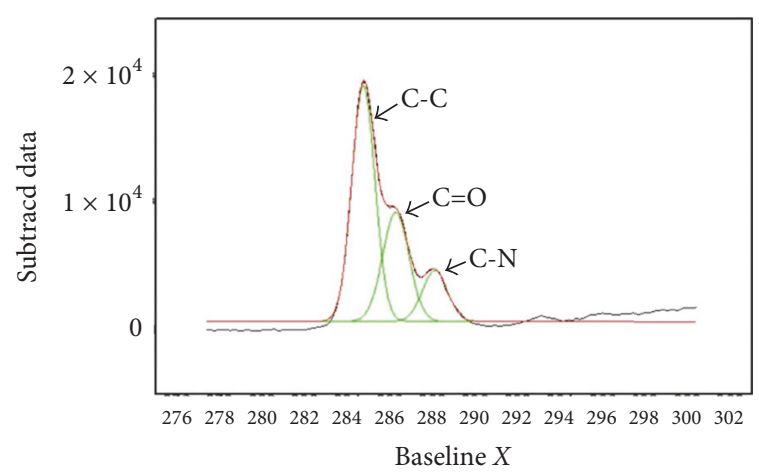

(b)

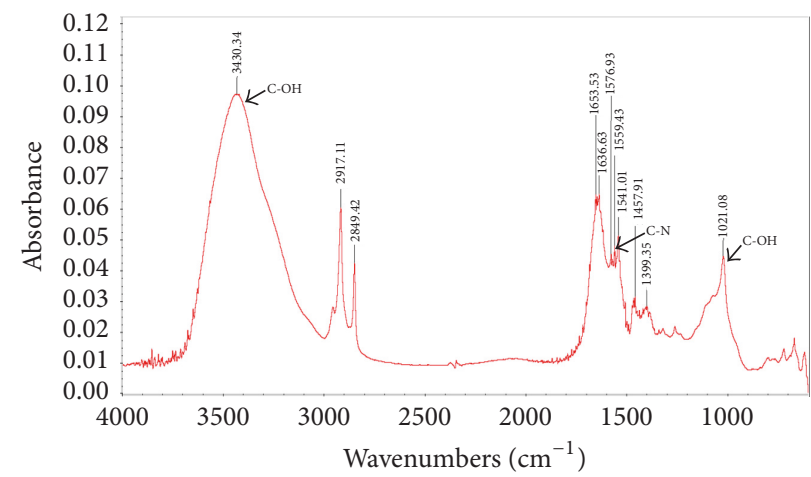

(c)

FIGURE 3: XPS and FTIR spectra and zeta potential. (a) The element detection. (b) XPS C1s spectrum. (c) FTIR spectra of as-prepared C-dots.

TABLE 1: X-ray photoelectron spectroscopy (XPS) spectra of the C-dots.

\begin{tabular}{|c|c|c|c|c|c|c|}
\hline Name & Start BE & Peak BE & End BE & Height & CPS area (P) CPS.eV & Atomic $\%$ \\
\hline C1s & $2.9 \times 10^{2}$ & $2.8 \times 10^{2}$ & $2.8 \times 10^{2}$ & $8.4 \times 10^{4}$ & $3.5 \times 10^{5}$ & 60.8 \\
\hline O1s & $5.4 \times 10^{2}$ & $5.3 \times 10^{2}$ & $5.2 \times 10^{2}$ & $1.0 \times 10^{5}$ & $3.6 \times 10^{5}$ & 25.0 \\
\hline N1s & 4 & $4 \times 10^{2}$ & $3.9 \times 10^{2}$ & $1.9 \times 10^{4}$ & $6.5 \times 10^{4}$ & 7.2 \\
\hline $\mathrm{Si} 2 \mathrm{p}$ & $1.1 \times 10^{2}$ & $1 \times 10^{2}$ & $0.9 \times 10^{2}$ & $8 \times 10^{3}$ & $2.5 \times 10^{4}$ & 4.6 \\
\hline Nals & $1.1 \times 10^{3}$ & $1.1 \times 10^{3}$ & $1.1 \times 10^{2}$ & $9.8 \times 10^{3}$ & $3.8 \times 10^{4}$ & 1.1 \\
\hline $\mathrm{Cl} 2 \mathrm{p}$ & $2 \times 10^{2}$ & $2 \times 10^{2}$ & $1.9 \times 10^{2}$ & $1.4 \times 10^{3}$ & $7.8 \times 10^{3}$ & 0.5 \\
\hline $\mathrm{P} 2 \mathrm{p}$ & $1.4 \times 10^{2}$ & $1.3 \times 10^{2}$ & $1.3 \times 10^{2}$ & $1.7 \times 10^{3}$ & $6 \times 10^{3}$ & 0.7 \\
\hline $\mathrm{C} 1 \mathrm{~s}$ & $3 \times 10^{2}$ & $2.8 \times 10^{2}$ & $2.8 \times 10^{2}$ & $2 \times 10^{4}$ & $5 \times 10^{4}$ & 0 \\
\hline
\end{tabular}

fluorescence signal. As shown in Figure 5, the CD labeled zebrafish clearly showed enhanced luminescence compared with its control. Fluorescence spectra analysis confirmed that the fluorescence signal came from the passivation of CDs in zebrafish. This result indicates that the passivation of CDs may have potential for imaging of vertebrate animals.

\section{Conclusions}

In conclusion, through simulating human gastrointestinal absorption digestion process, we successfully synthesized highly fluorescence and biological activated carbon using gastric and intestinal juice to simply process traditional carbon black borneol. These CDs show multicolor fluorescence, excellent light stability, and their suitability used in various
$\mathrm{pH}$ conditions. The black synthetic borneol, the dry stool carbonization of wild boars belonging to pig families, is one of the typical carbonized Mongolian medicines with the effects of invigorating the stomach and promoting the digestion and so forth. The black synthetic borneol also named Har Gabur in Mongolian is the most distinctive carbon herbs for the treatment of gastrointestinal diseases and can be mixed with other drugs used in a variety of formulations. So far, a lot of black borneol function was not clear. It needs more in-depth study and exploration.

Through the literature research we found that the current researchers have developed a variety of preparation methods of CDs, such as synthetic method, electrochemical method, laser ablation method, acid dissolution method, and hydrothermal method, in which CDs are synthesized 

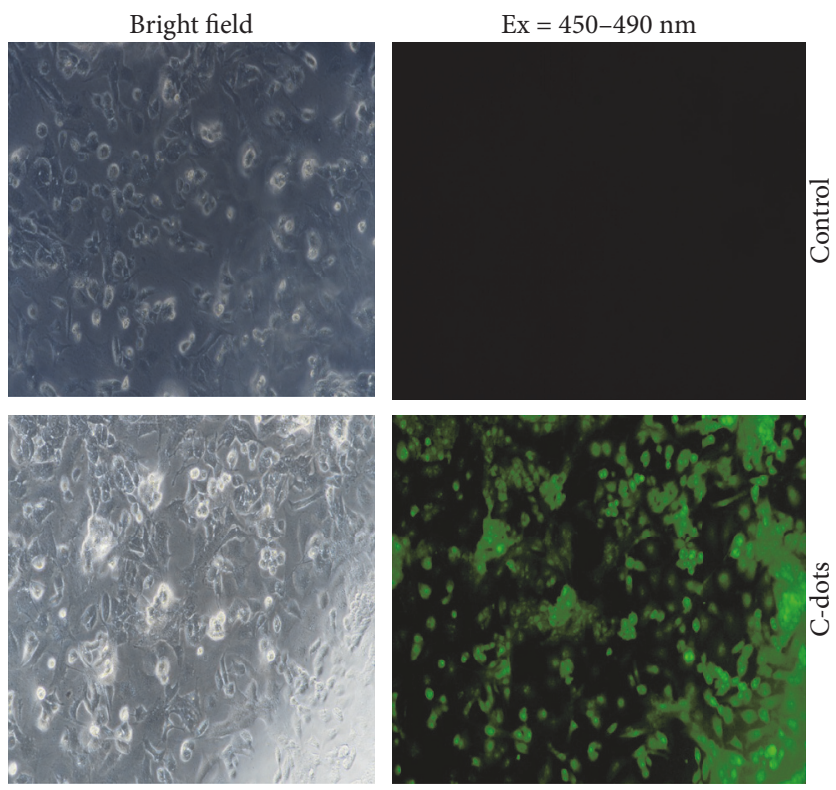

$\overline{50 \mu \mathrm{m}}$

Figure 4: Bright filed and fluorescence microphotographs of HepG2 cells incubated with PLL-passivated C-dots (C-dots) and without C-dots (Control) with an excitation filter of 450-490 $\mathrm{nm}$ for green color, respectively.

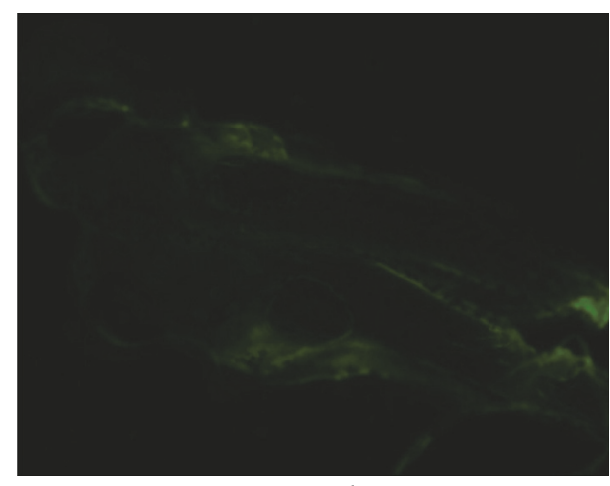

Control

(a)

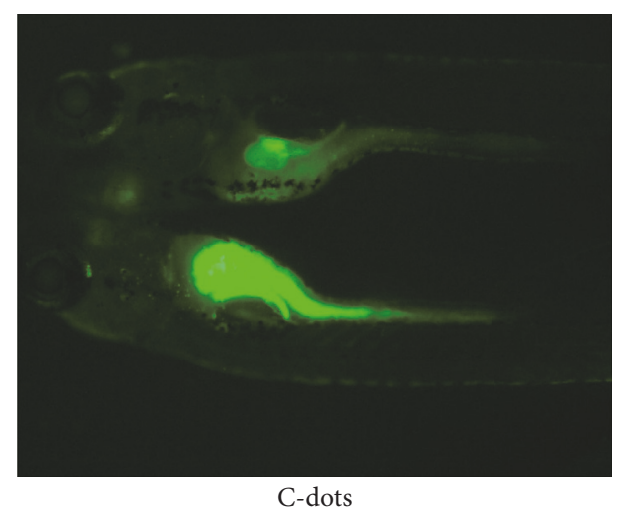

(b)

Figure 5: Ex vivo zebrafish imaging. Photograph of the C-dot labeled zebrafish (C-dots) and its control (control) (a) and fluorescence image (b) of the C-dot labeled zebrafish, its control, and bare C-dots measured with fluorescence microscope imaging system. Fluorescence spectra comparison of the C-dots and their control.

using physical and chemical methods at the great costs of labor force and money. However, an important preparation method, enzyme digestion method, has not yet been applied to the preparation of CDs. More importantly, there is close relationship between this method and traditional medicine digestion in the human body and carbon absorption in the human body. Thus prepared CDs completely dissolve in the human body without any security risks and even do good to improve immunity and have other health effects.

In enzyme digestion method, Har Gabur was processed by intestinal fluid to prepare CDs. Following the heat treatment in $37^{\circ} \mathrm{C}$ water, pepsin or trypsin will first attack the defect area of the surface of the carbon particles, "cutting" or "dividing" those carbon particles, forming the smaller size quantum dots, ice sheet fluorescent CDs.

The surface of ice sheet fluorescent CDs prepared by such method is rich in amino functional organic groups, which makes it not only have good dispersibility, water solubility, easy functionalization, and biological coupling markers but also have good biocompatibility. We will model ice sheet fluorescent nano-CDs to explore the relationship between chemical properties and biological properties of nanomaterials in the field of cells. Choosing the most suitable carbon for preparation and biological safety detection is also our next plan. It will help to carry out the new fluorescent nanomaterials development and application of optical imaging studies. Ice sheet fluorescent CDs provide 
valuable information for optical imaging in tumor diagnosis, especially early diagnosis and imaging research.

\section{Conflicts of Interest}

The authors declare that they have no conflicts of interest.

\section{Authors' Contributions}

Jun Ai provided technical support.

\section{Acknowledgments}

This work was supported by the project "The Innovative Research Team of the New Drug Research and the Industrialization of Mongolian Medicine" of "Prairie Talents" Engineering, no. NEI ZU TONG ZI[2015]56, and the Program of Hospital Project of Inner Mongolia International Mongolian Hospital (Grant no. 2016YJS22).

\section{References}

[1] M. Tan, L. Zhang, R. Tang et al., "Enhanced photoluminescence and characterization of multicolor carbon dots using plant soot as a carbon source," Talanta, vol. 115, pp. 950-956, 2013.

[2] C.-L. Li, C.-M. Ou, C.-C. Huang et al., "Carbon dots prepared from ginger exhibiting efficient inhibition of human hepatocellular carcinoma cells," Journal of Materials Chemistry B, vol. 2, no. 28, pp. 4564-4571, 2014.

[3] H. Wang, C. Sun, X. Chen et al., "Excitation wavelength independent visible color emission of carbon dots," Nanoscale, vol. 9, no. 5, pp. 1909-1915, 2017.

[4] K. Yang, S. Wang, Y. Wang, H. Miao, and X. Yang, "Dualchannel probe of carbon dots cooperating with gold nanoclusters employed for assaying multiple targets," Biosensors and Bioelectronics, vol. 91, no. 15, pp. 566-573, 2017.

[5] Y. Yeon, M. Y. Lee, S. Y. Kim et al., "Production of quasi-2D graphene nanosheets through the solvent exfoliation of pitchbased carbon fiber," Nanotechnology, vol. 26, no. 37, Article ID 375602, 2015.

[6] Q. Wang, C. Zhang, G. Shen, H. Liu, H. Fu, and D. Cui, "Fluorescent carbon dots as an efficient siRNA nanocarrier for its interference therapy in gastric cancer cells," Journal of Nanobiotechnology, vol. 12, no. 1, article 58, 2014.

[7] H. Liu, Q. Wang, G. Shen et al., "A multifunctional ribonuclease A-conjugated carbon dot cluster nanosystem for synchronous cancer imaging and therapy," Nanoscale Research Letters, vol. 9, no. 1, pp. 1-11, 2014.

[8] X. Yang, Y. Wang, X. Shen et al., "One-step synthesis of photoluminescent carbon dots with excitation-independent emission for selective bioimaging and gene delivery," Journal of Colloid and Interface Science, vol. 492, pp. 1-7, 2017.

[9] P. Namdari, B. Negahdari, and A. Eatemadi, "Synthesis, properties and biomedical applications of carbon-based quantum dots: an updated review," Biomedicine \& Pharmacotherapy, vol. 87, pp. 209-222, 2017.

[10] A. S. Minin, A. V. Belousova, L. T. Smolyuk, M. V. Ulitko, M. A. Uimin, and I. V. Byzov, "Interactions of bimodal magnetic and fluorescent nanoparticles based on carbon quantum dots and iron-carbon nanocomposites with cell cultures," Bulletin of
Experimental Biology and Medicine, vol. 162, no. 2, pp. 248-251, 2016.

[11] J. Wang, C.-F. Wang, and S. Chen, "Amphiphilic eggderived carbon dots: rapid plasma fabrication, pyrolysis process, and multicolor printing patterns," Angewandte Chemie-International Edition, vol. 51, no. 37, pp. 9297-9301, 2012.

[12] J. Fan and P. K. Chu, "Group IV nanoparticles: Synthesis, properties, and biological applications," Small, vol. 6, no. 19, pp. 2080-2098, 2010.

[13] W. Li, Z. Zhang, B. Kong et al., "Simple and green synthesis of nitrogen-doped photoluminescent carbonaceous nanospheres for bioimaging," Angewandte Chemie - International Edition, vol. 52, no. 31, pp. 8151-8155, 2013.

[14] H. Liu, T. Ye, and C. Mao, "Fluorescent carbon nanoparticles derived from candle soot," Angewandte Chemie International Edition, vol. 46, no. 34, pp. 6473-6475, 2007.

[15] C. Wan, Z. Bao, L. Que, N. Song, and Z. G. Tang, "The effect of piper longum. L on blood pressure and body weight in insulin resistance syndrome rats," Journal of Medicine \& Pharmacy of Chinese Minorities, vol. 5, no. 5, pp. 23-26, 2009.

[16] M. Yuan, R. Zhong, H. Gao et al., "One-step, green, and economic synthesis of water-soluble photoluminescent carbon dots by hydrothermal treatment of wheat straw, and their bioapplications in labeling, imaging, and sensing," Applied Surface Science, vol. 355, pp. 1136-1144, 2015.

[17] J. Ai, J. Li, L. Ga, G. Yun, L. Xu, and E. Wang, "Gquadruplex/protoporphyrin IX-functionalized silver nanoconjugates for targeted cancer cell photodynamic therapy," RSC Advances, vol. 6, no. 99, pp. 96942-96945, 2016.

[18] J. Ai, J. Li, Ga. Lu, GH. Yun, and Li. Xu, "Multifunctional nearinfrared fluorescent nanoclusters forsimultaneous targeted cancer imaging and photodynamic therapy," Sensors Actuators B Chemical, pp. 222-918, 2015.

[19] W. Li, W. Zhang, X. Dong et al., "Porous heterogeneous organic photocatalyst prepared by HIPE polymerization for oxidation of sulfides under visible light," Journal of Materials Chemistry, vol. 22, no. 34, pp. 17445-17448, 2012.

[20] W. Li, L. Li, H. Xiao et al., "Iodo-BODIPY: A visible-lightdriven, highly efficient and photostable metal-free organic photocatalyst," RSC Advances, vol. 3, no. 32, pp. 13417-13421, 2013.

[21] W. Li, M. Jiang, Y. Cao et al., "Turning ineffective transplatin into a highly potent anticancer drug via a prodrug strategy for drug delivery and inhibiting cisplatin drug resistance," Bioconjugate Chemistry, vol. 27, no. 8, pp. 1802-1806, 2016.

[22] R. Du, H. Xiao, G. Guo et al., "Nanoparticle delivery of photosensitive Pt(IV) drugs for circumventing cisplatin cellular pathway and on-demand drug release," Colloids and Surfaces B: Biointerfaces, vol. 123, pp. 734-741, 2014. 

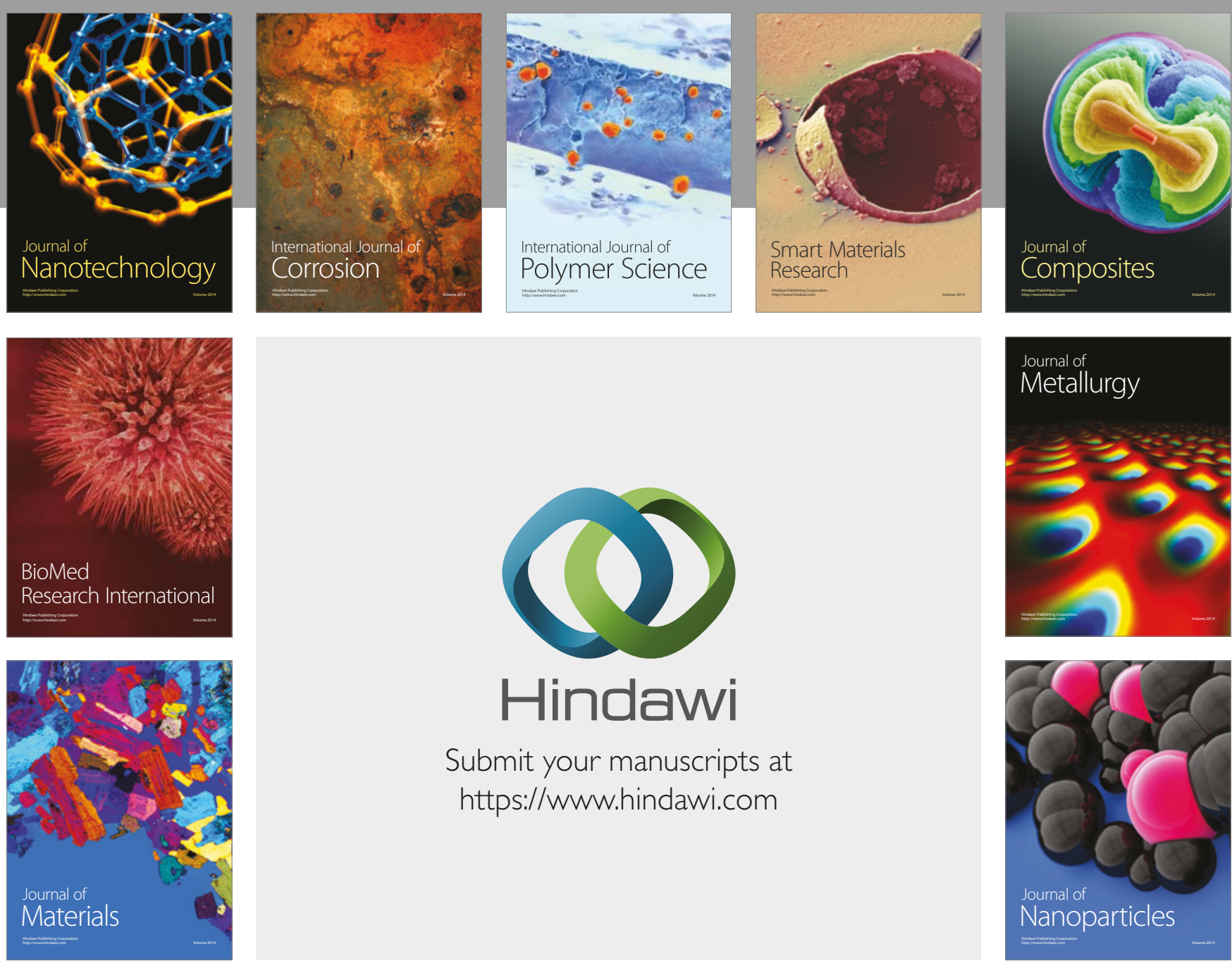

\section{Hindawi}

Submit your manuscripts at

https://www.hindawi.com
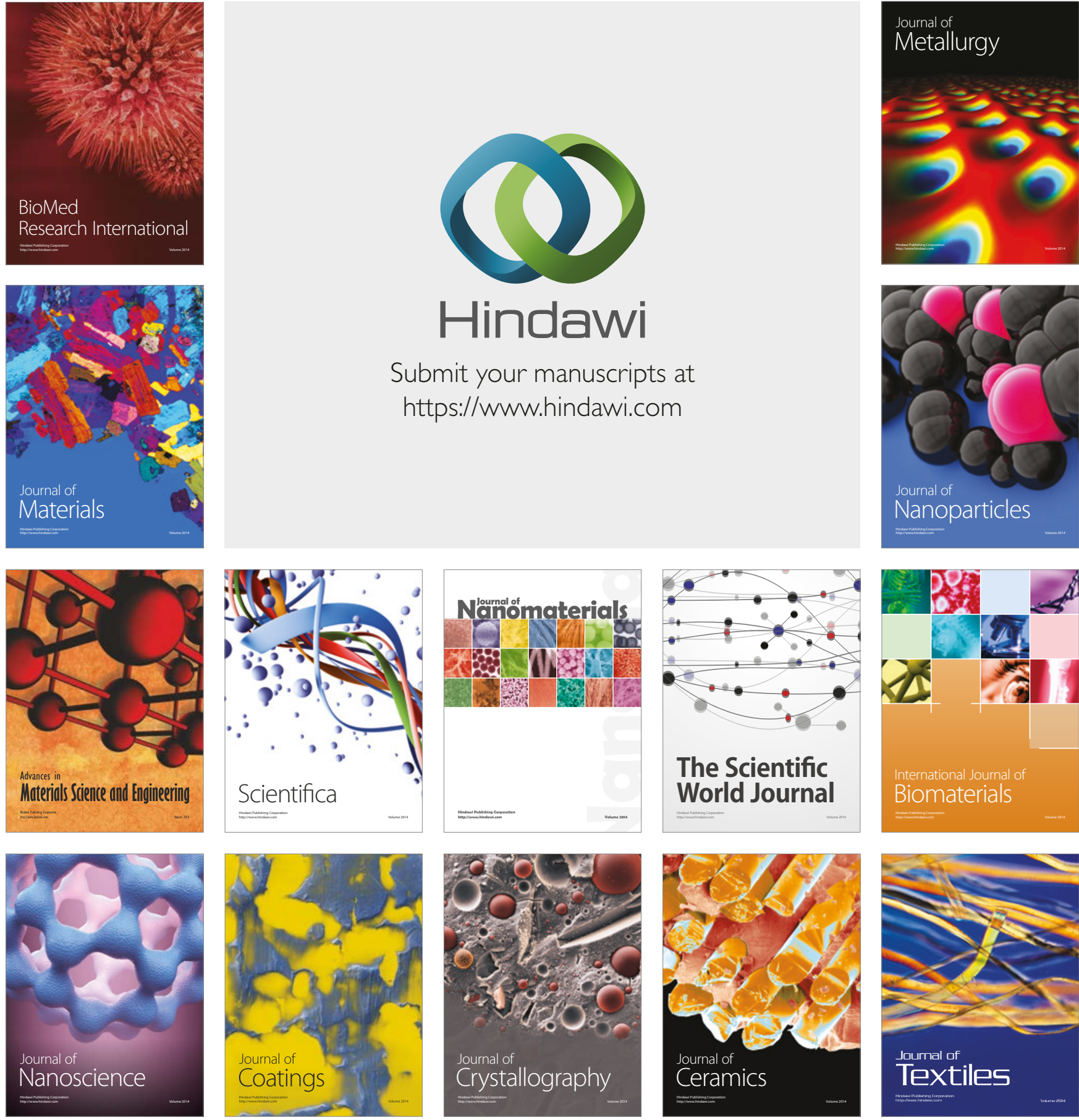

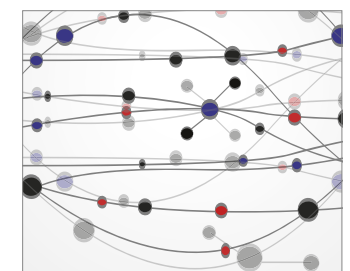

The Scientific World Journal
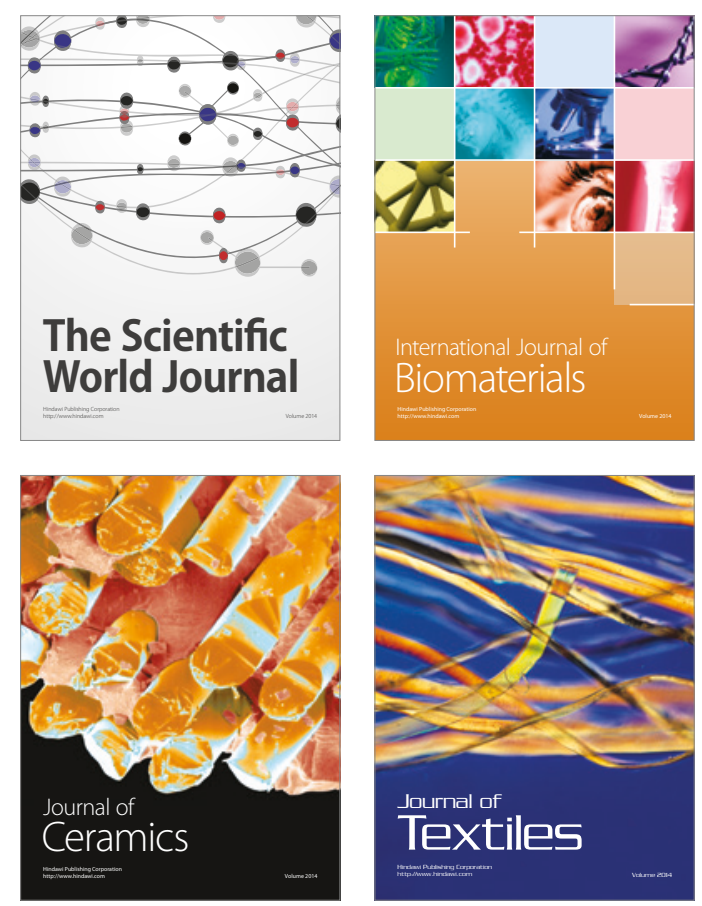